\title{
Motor Unit Number Estimation and Motor Unit Action Potential Analysis in Carpal Tunnel Syndrome
}

\author{
Min Kyun Sohn, M.D., Sung Ju Jee, M.D., Seon Lyul Hwang, M.D., \\ Young-Jae Kim, M.D., Hyun-Dae Shin, M.D. ${ }^{1}$ \\ Departments of Rehabilitation Medicine, ${ }^{1}$ Orthopedic Surgery, School of Medicine, \\ Chungnam National University, Daejeon 301-172, Korea
}

Objective To evaluate the clinical significance of motor unit number estimation (MUNE) and quantitative analysis of motor unit action potential (MUAP) in carpal tunnel syndrome (CTS) according to electrophysiologic severity, ultrasonographic measurement and clinical symptoms.

Method We evaluated 78 wrists of 45 patients, who had been diagnosed with CTS and 42 wrists of 21 healthy controls. Median nerve conduction studies, amplitude and duration of MUAP, and the MUNE of the abductor pollicis brevis were measured. The cross sectional area (CSA) of the median nerve at the pisiform and distal radioulnar joint level was determined by high resolution ultrasonography. Clinical symptom of CTS was assessed using the Boston Carpal Tunnel Questionnaire (BCTQ).

Results The MUNE, the amplitude and the duration of MUAP of the CTS group were significantly different from those found in the control group. The area under the ROC curve was 0.944 for MUNE, 0.923 for MUAP amplitude and 0.953 for MUAP duration. MUNE had a negative correlation with electrophysiologic stage of CTS, amplitude and duration of MUAP, CSA at pisiform level, and the score of BCTQ. The amplitude and duration of MUAP had a positive correlation with the score of BCTQ. The electrophysiologic stage was correlated with amplitude but not with the duration of MUAP.

Conclusion MUNE, amplitude and duration of MUAP are useful tests for diagnosis of CTS. In addition, the MUNE serves as a good indicator of CTS severity.

Key Words Carpal tunnel syndrome, Electrodiagnosis, Electromyography, Ultrasonography

Received April 18, 2011; Accepted October 9, 2011

Corresponding author: Seon Lyul Hwang

Department of Rehabilitation Medicine, Chungnam National University Hospital, Munhwa-ro 282, Jung-gu, Daejeon 301-172, Korea

Tel: +82-42-280-7811, Fax: +82-42-256-6056, E-mail: h5034@cnuh.co.kr (c) This is an open-access article distributed under the terms of the Creative Commons Attribution Non-Commercial License (http:// creativecommons.org/licenses/by-nc/3.0) which permits unrestricted noncommercial use, distribution, and reproduction in any medium, provided the original work is properly cited.

Copyright $\odot 2011$ by Korean Academy of Rehabilitation Medicine

\section{INTRODUCTION}

Carpal tunnel syndrom (CTS) is a peripheral neuropathy caused by median nerve compression at the wrist causing pain, paresthesia, and functional problems. The pathophysiology of CTS is characterized by nerve demyelination in early stages and axonal degeneration in the later stages. In CTS, electrodiagnostic methods are used to evaluate the degree of focal demyelination and 
axonal degeneration. ${ }^{1}$ Stevens ${ }^{2}$ asserted that the main mechanism for mild CTS was the conduction block or delay and axonotmesis in moderate to severe CTS. The decrease in axon number of resulting from axonotmesis decreases the amplitude of compound muscle action potential (CMAP). However, when that amplitude was retained due to collateral sprouting, the motor unit number estimation (MUNE) could be used to estimate the degree of axonal degeneration. ${ }^{3}$ MUNE quantifies functional motor units; it has been reported that motor units decrease in CTS. ${ }^{4-7}$ Bayrak et al. ${ }^{7}$ reported greater MUNE decreases in increasing electrophysiological severity and cross sectional area of median nerve by and ultrasonography.

Motor unit action potential (MUAP) analysis provides information on fundamental pathology of the neuromuscular disease, and extended and accurate information on muscle. ${ }^{8}$ In neuropathies, nerve regeneration increases nerve fibers and their density that leads to increased amplitude, duration, and the phases of MUAP. ${ }^{9}$ The needle electromyography of the abductor pollicis brevis (APB) of CTS patients is used to evaluate the severity of axonal degeneration by the degree of abnormal spontaneous activity. Cuturic and Palliyath ${ }^{10}$ reported that CTS patients with polyphasic MUAP in APB showed lower MUNE compare to the control group. Werner and Albers ${ }^{11}$ reported that patient group with increased MUAP amplitude showed delayed latency and decreased amplitude of the median motor and sensory nerve conduction studies. However, there were no studies on the change or relationship of MUAP based on electrophysiologic or clinical severity of CTS.

Therefore, we evaluated the differences of MUNE, amplitude and duration of MUAP at APB between CTS patients and the control group. In addition, we analyzed the relationship between median nerve conduction studies, clinical scales, and median nerve ultrasonographic findings in CTS.

\section{MATERIALS AND METHODS}

\section{Subjects}

From April 2009 to March 2010, 78 hands of 45 subjects were diagnosed with CTS through the use of electrodiagnostic testing. These patients had presented with pain, paresthesia, and numbness in the median nerve distribution areas, weakness of the APB, and positive findings in provocation tests, such as the Tinel test or the Phalen maneuver. The mean age of the patient group was $59.2 \pm 10.3$ years. There were 36 females and 9 males in the patient group. Bilateral CTS was observed in 33 subjects and unilateral CTS in 12 subjects. The average duration of disease had been $15 \pm 26$ months. The control group was comprised of 21 subjects having a mean age of $51.8 \pm 10.2$ years; there were 16 females and 5 males in the control group. The electrodiagnostic diagnosis of CTS requires one or more of the following 4 criterion: median distal motor latency longer than $4.3 \mathrm{~ms}$, median distal sensory latency longer than $3.5 \mathrm{~ms}$, median sensory latency difference between the latency of wrist stimulation and palm stimulation more than $1.6 \mathrm{~ms}$, and latency difference of median and ulnar sensory nerve recording from ring finger more than $0.6 \mathrm{~ms} .^{12}$

We excluded subjects with any of the following: predisposing causes for CTS, a previous history of treatment of CTS, history of fracture or surgery in the wrist, or any other neuropathy such as peripheral neuropathy or cervical radiculopathy.

\section{Methods}

Electrophysiologic evaluation: Electrodiagnostic tests were performed with Keypoint (Dantec, Skovlunde, Denmark) to evaluate for motor and sensory nerve conduction, MUNE, and quantitative analysis of MUAP. Room temperature was kept between $26-28^{\circ} \mathrm{C}$.

Nerve conduction study: The median motor nerve conduction study was performed by supramaximal electrical stimulation of the median nerve at the wrist and recording from an active electrode placed over the motor point of the APB muscle $8 \mathrm{~cm}$ from the stimulation point with the reference electrode over the metacarpophalangeal (MCP) joint of the thumb. Distal motor latency and baseline-negative peak amplitude of CMAPs of median nerve were measured. Sensory nerve conduction study was performed by positioning the recording electrode on the index finger and stimulating the proximal median nerve at points $7 \mathrm{~cm}$ and $14 \mathrm{~cm}$ from the recording electrode. Through these tests, distal sensory latency, baseline-negative peak amplitude of sensory nerve action potentials (SNAPs), and conduction velocity at the wrist segment were measured. With the above data as reference, electrophysiologic severity of 
CTS was classified using the Padua scale (Appendix 1). ${ }^{13}$ MUNE test: MUNE test was performed using a manual incremental method. MUNE was recorded from a surface active recording electrode placed over the motor point of the APB with the reference over the MCP joint of the thumb. The median nerve was stimulated at wrist by bar electrode, at $8 \mathrm{~cm}$ proximal to the active electrode. For accurate nerve stimulation, a bar electrode was fixated at wrist prior to stimulation.

A built-in MUNE analysis program in the electrophysiologic instrument was used and the maximum $\mathrm{M}$ response was first obtained by increasing stimulation intensity. Next, $\mathrm{M}$ responses at threshold intensity were obtained by reducing the stimulation intensity, and stages involving recording the minimal increases of $\mathrm{M}$ responses through the minimal increase of stimulation intensity were repeated. A total of 11 stages were recorded and MUNE was calculated using formula given below.

\section{Maximum M area}

Motor units number=

(Maximum $\mathrm{M}$ area - Initial $\mathrm{M}$ area) / stages

Quantitative MUAP analysis: Quantitative MUAP analysis was performed using built-in multi MUP program. After inserting the monopolar needle electrode (TECA, 37 $\mathrm{mm}$ ) into the mid-portion of the APB, the needle position was adjusted to place the electrode near the contracting muscle fiber. With the subject maintaining a minimal muscle contraction for 5-10 sec, the multi MUP program recorded up to 6 MUAPs at once with filtered frequency of $5 \mathrm{~Hz}-10 \mathrm{kHz}$, amplitude more than $50 \mu \mathrm{V}$ and slope from initial negative peak to positive peak more than $30 \mu \mathrm{V} / 0.1$ ms. ${ }^{14}$ Amplitude and duration of 20 different MUAPs were recorded over 3 to 5 insertions. The amplitude of MUAP was defined as the difference in the potential between the maximum negative peak to the maximum positive peak. The duration of MUAP was defined as the period between the initial departure and the final return to the baseline.

Ultrasonography examination: Ultrasonography examination with the Envisor Ultrasound system (Philips, Bothell, USA) measured the cross-sectional area (CSA) of median nerve. All measurements were performed on both hands of the subjects by the same physician; the electrophysiologic results were masked during this measurement. During the examination, patients were seated in a comfortable position with the hands placed on a hard surface, elbows flexed, and forearms in supination position. Neither external pressure nor the weight of the probe was applied to avoid causing any artificial nerve deformity. The median nerve was examined axially at the levels of the distal radioulnar joint and the pisiform. The CSA was measured by utilizing a method of tracing a continuous line around the surrounding hyperechogenic rim of the myelin.

Clinical scales: The clinical scales of the CTS were assessed using the Boston carpal tunnel questionnaire (BCTQ). ${ }^{15}$ This was comprised of 11 questions on symptoms and 8 questions on functional status. The symptom severity scale consisted of responses numbered 1 (no symptoms) to 5 (most severe). Functional status scale consisted of responses numbered 1 (performance with no difficulty) to 5 (unable to perform due to symptoms). The BCTQ was self-completed by patients and the physician recorded the total score.

Statistical analysis: Statistical analysis was performed using the Korean-language version of SPSS 12.0. Each results of the patient and control groups were verified though independent $t$-tests. The correlation between variables of the CTS patient was compared using Pearson's correlation test. The comparison of Padua scales of each group was performed with oneway ANOVA and p value less than 0.05 was considered statistically significant. Also the receiver operating characteristic (ROC) curve was used to compare the diagnostic usefulness of the amplitude and the duration of MUAP, and MUNE.

\section{RESULTS}

\section{Comparison of patient and control group}

MUNE of the patient group was 81.8 \pm 33.9 and statistically significantly lower than MUNE of the control group, which was $136.4 \pm 22.0(\mathrm{p}<0.05)$. The amplitude of the MUAP in the patient and the control group was $1,539.1 \pm 625.1 \mu \mathrm{V}$ and $871.7 \pm 192.7 \mu \mathrm{V}$, respectively; the duration of the MUAP in the patient and the control group was $14.3 \pm 2.91 \mathrm{~ms}$ and $10.1 \pm 1.74 \mathrm{~ms}$, respectively. The amplitude and the duration were both significantly increased in the patient group $(\mathrm{p}<0.05)$.

The CSA of the median nerve of the patient group at the pisiform and distal radioulnar joint level was 
$13.1 \pm 3.2 \mathrm{~mm}^{2}$ and $11.6 \pm 2.7 \mathrm{~mm}^{2}$, respectively, which was significantly increased compared to the CSA of the control group which was $10.8 \pm 1.7 \mathrm{~mm}^{2}$ and $10.4 \pm 2.1 \mathrm{~mm}^{2}$ at the pisiform and distal radioulnar level, respectively $(\mathrm{p}<0.05)$ (Fig. 1).

Diagnostic significance of MUNE and amplitude and duration of MUAP

The area under the curve (AUC) of the ROC curve

A

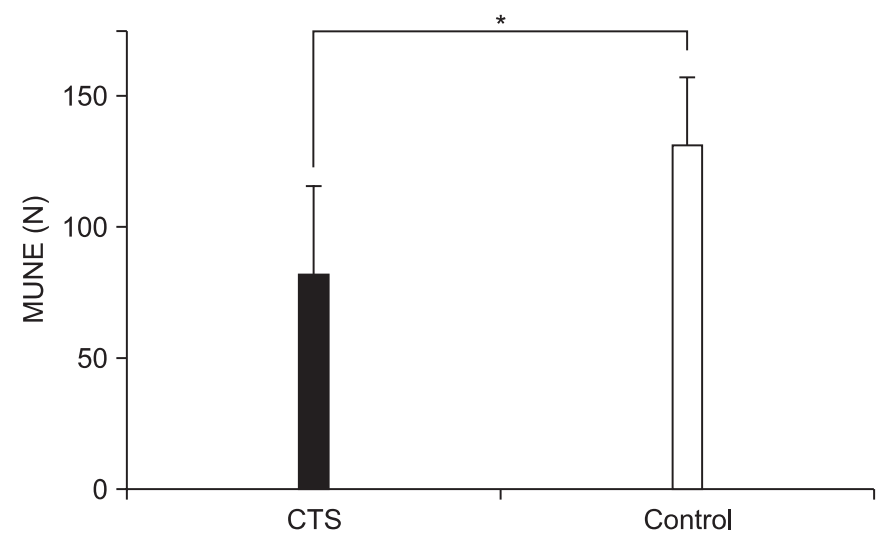

C

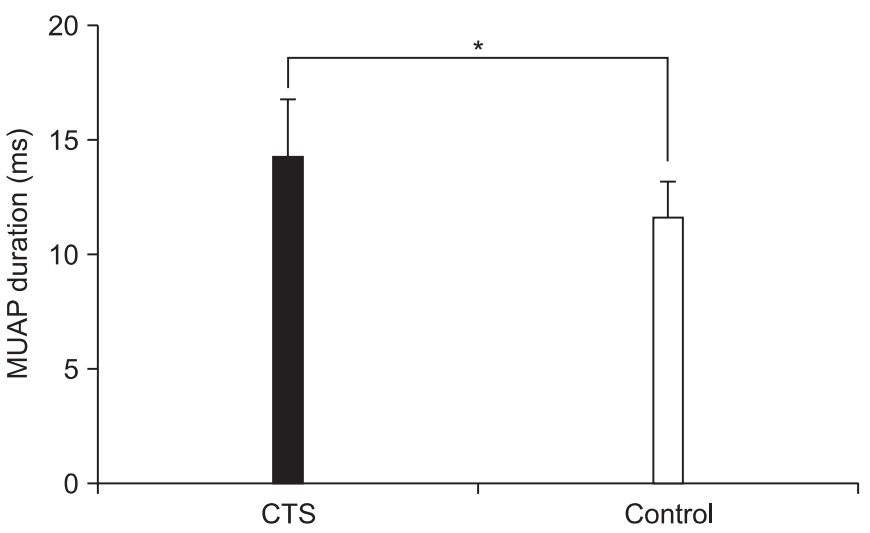

E

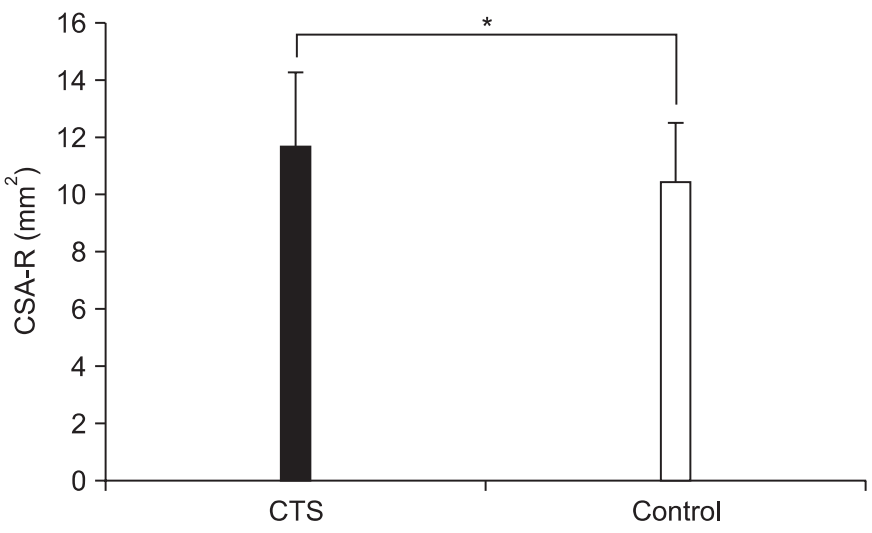

showed 0.944 for MUNE, 0.923 for MUAP amplitude, and 0.953 for MUAP duration in the $95 \%$ confidence interval, respectively (Fig. 2). High specificity and sensitivity were shown with the following cut-off values for CTS diagnostic criteria based on ROC values: 112.55 for MUNE (sensitivity of $96.7 \%$, specificity of $85.9 \%$ ), 923.5 $\mu \mathrm{V}$ for MUAP amplitude (sensitivity of $87.2 \%$, specificity of $80 \%$ ), and 10.6 ms for MUAP duration (sensitivity of $92.3 \%$, specificity of $80 \%$ ).

B

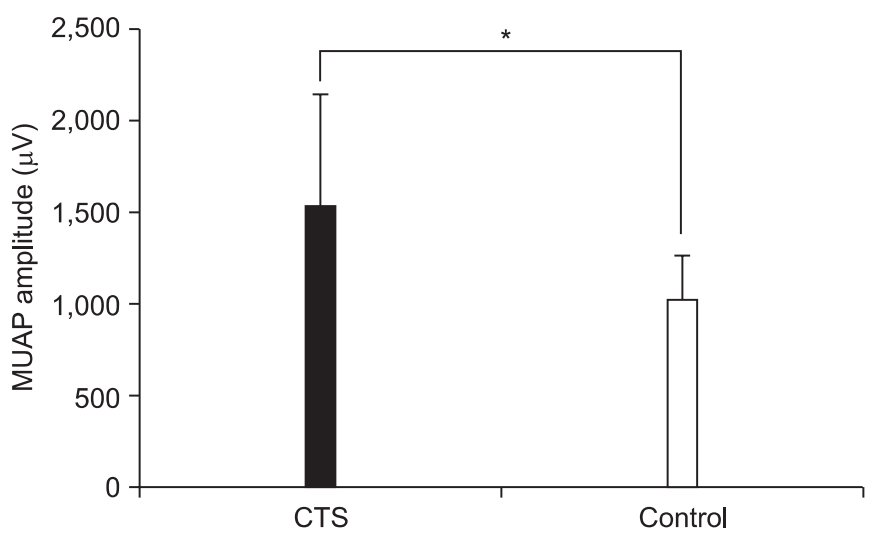

D

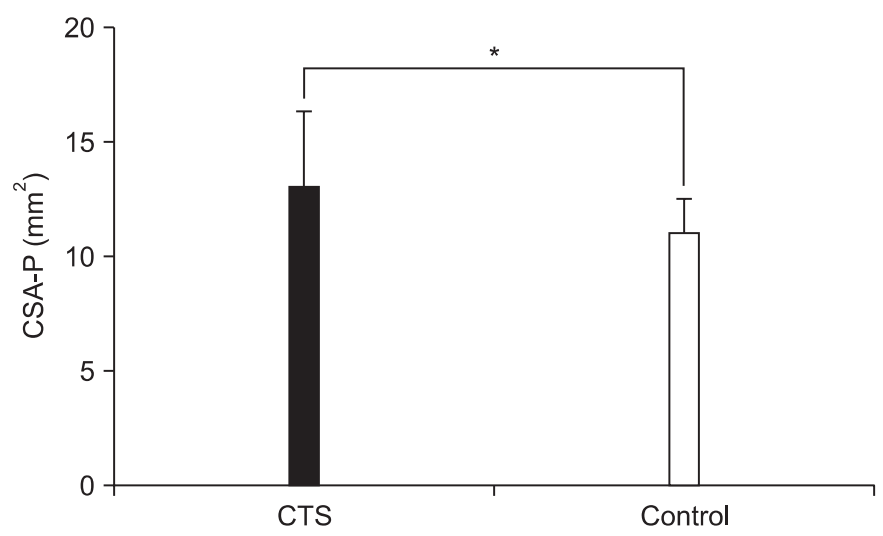

Fig. 1. The MUNE (A), MUAP amplitude (B) and duration (C) of APB and cross-sectional area of median nerve at pisiform (D) and radio-ulnar joint level (E) showed statistically significant differences between the CTS and control group ( ${ }^{*} \mathrm{p}<0.05$ by independent $\mathrm{t}$-test). 
A

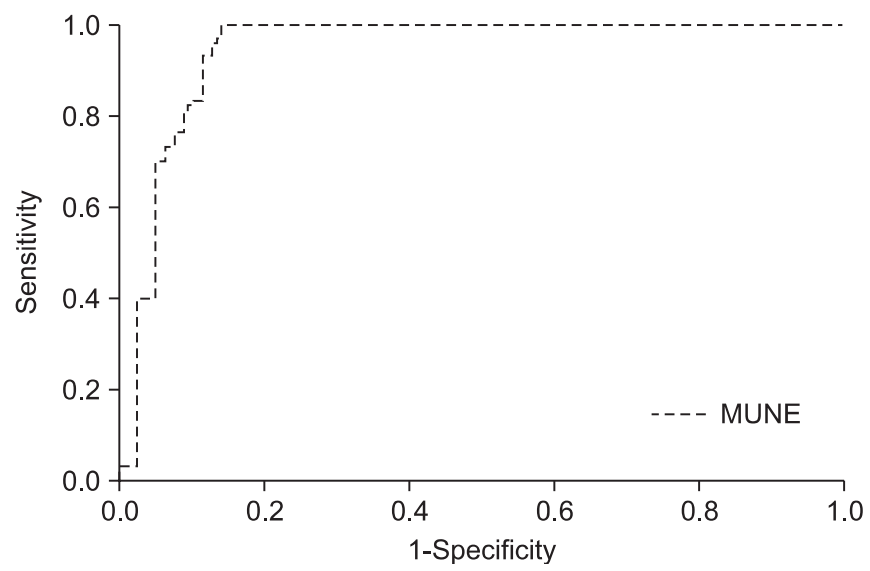

B

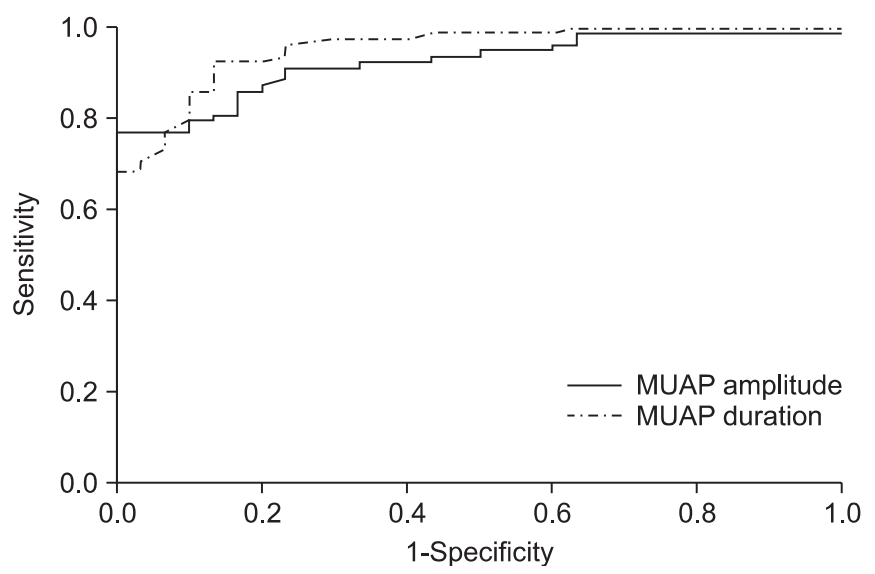

Fig. 2. Receiver operating characteristic (ROC) curves of MUNE (A), MUAP amplitude and duration (B) of APB. The area under the curve of MUNE is 0.944, MUAP amplitude is 0.923, MUAP duration is $0.953(\mathrm{p}<0.05)$.

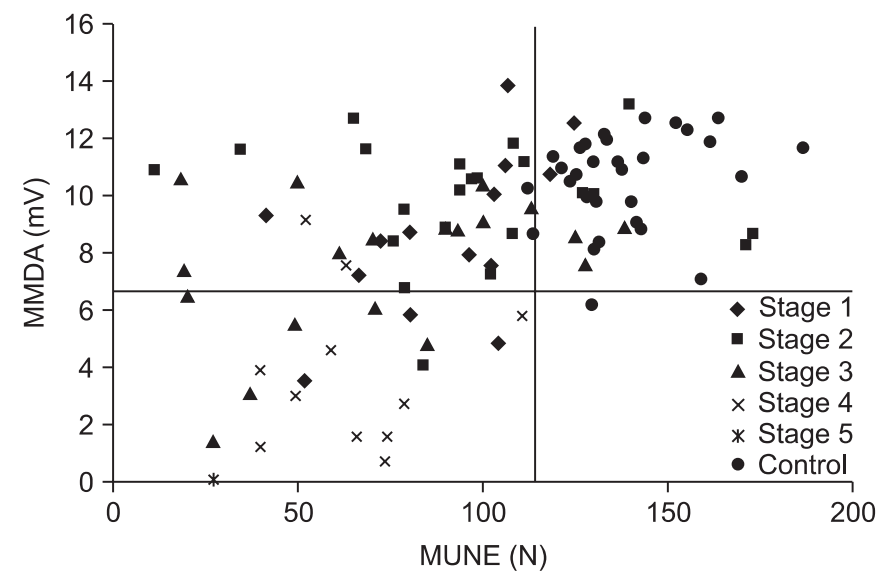

Fig. 3. The MUNE and median motor distal amplitude (MMDA) of APB were compared in a patient with CTS according to the electrophysiologic severity and control groups. The abnormal cutoff values were 112.55 for MUNE and $6.35 \mathrm{mV}$ for MMDA.

\section{Comparison of MUNE and median motor distal} amplitude

With cut-off values to diagnose CTS as 112.55 for MUNE and $6.35 \mathrm{mV}$ for median motor distal amplitude, many patients showed low MUNE with normal range of median motor distal amplitude. This tendency was observed in 1-4 stages of Padua, regardless of electrophysiologic severity. However, low median motor distal amplitude was not observed in patients with a MUNE in the normal range (Fig. 3).
Difference in CTS patients by electrophysiologic severity MUNE decreased with statistical significance with increasing Padua stages $(\mathrm{F}=5.064, \mathrm{p}<0.001)$. And MUNE showed statistically significant decrease in Padua stage 4 and 5 in comparison to Padua stage $1(\mathrm{p}<0.05)$. Amplitude of MUAP increased with statistical significance with increasing Padua stages $(\mathrm{F}=2.909, \mathrm{p}=0.027)$, but multiple comparisons showed statistically significant increase in Padua stage 3 compared to stage $1(p<0.05)$. There were no significant changes in the duration of MUAP according to Padua stages.

CSA of median nerve showed significant increase at pisiform level $(\mathrm{F}=3.9454, \mathrm{p}=0.006)$ and distal radioulnar joint level $(\mathrm{F}=3.408, \mathrm{p}<0.014)$ with increased electrophysiological severity. Especially, the CSA at pisiform level was increased with statistical significance in Padua stage 4 and 5 compared to stage $1(\mathrm{p}<0.05)$ (Table 1$)$.

\section{Correlation between tests}

MUNE of the APB showed statistically significant positive correlation with median motor distal amplitude ( $\mathrm{r}=0.402, \mathrm{p}=0.001)$, amplitude of SNAP ( $\mathrm{r}=0.353, \mathrm{p}=0.002)$, and median sensory nerve conduction velocity at carpal tunnel ( $\mathrm{r}=0.240, \mathrm{p}=0.038$ ), and statistically significant negative correlation with CSA at pisiform level $(\mathrm{r}=-0.327$, $\mathrm{p}=0.007)$, distal latency of CMAP $(\mathrm{r}=-0.428, \mathrm{p}=0.001)$, MUAP amplitude $(\mathrm{r}=-0.255, \mathrm{p}=0.024)$ and duration $(\mathrm{r}=-0.273, \mathrm{p}=0.016)$, and total scores of BCTQ $(\mathrm{r}=-0.483$, $\mathrm{p}=0.001)$, respectively $(\mathrm{p}<0.05)$. 
Table 1. MUNE, Amplitude and Duration of MUAP and Ultrasonographic Measurements According to Electrophysiologic Severity in CTS

\begin{tabular}{cccccc}
\hline Stage & MUNE & MUAP amplitude & MUAP duration & CSA-P & CSA-R \\
\hline 1 & $96.10 \pm 35.04$ & $1,270.56 \pm 488.74$ & $13.56 \pm 3.15$ & $11.66 \pm 2.17$ & $11.08 \pm 3.02$ \\
2 & $88.12 \pm 25.70$ & $1,509.47 \pm 488.12$ & $14.32 \pm 2.03$ & $13.22 \pm 2.81$ & $13.54^{*} \pm 3.14$ \\
3 & $85.66 \pm 35.14$ & $1,882.21^{*} \pm 769.50$ & $14.80 \pm 2.40$ & $12.66 \pm 2.54$ & $10.52 \pm 2.04$ \\
4 & $53.71^{*} \pm 20.61$ & $1,612.85 \pm 619.72$ & $15.22 \pm 1.94$ & $14.66^{*} \pm 3.72$ & $11.61 \pm 1.25$ \\
5 & $59.14^{*} \pm 32.18$ & $1,365.20 \pm 555.84$ & $13.68 \pm 2.79$ & $17.05^{*} \pm 5.44$ & $12.80 \pm 2.54$ \\
$P$ & 0.001 & 0.027 & 0.321 & 0.006 & 0.014 \\
F & 5.064 & 2.909 & 1.194 & 3.945 & 3.408 \\
\hline
\end{tabular}

The values are mean \pm standard deviation

CSA-P: Cross-sectional area of median nerve at pisiform level, CSA-R: Cross-sectional area of median nerve at distal radioulnar joint level

${ }^{*} \mathrm{p}<0.05$ compared to stage 1

Table 2. Correlation Coefficients between Electrophysiologic results and Ultrasonographic Measurements and Clinical Scale

\begin{tabular}{|c|c|c|c|c|c|c|c|c|c|c|c|}
\hline & MUNE & CSA-P & CSA-R & MMDL & MMDA & MSDL & MSDA & SCV & $\begin{array}{c}\text { MUAP } \\
\text { amplitude }\end{array}$ & $\begin{array}{c}\text { MUAP } \\
\text { duration }\end{array}$ & BCTQ \\
\hline MUNE & 1 & & & & & & & & & & \\
\hline CSA-P & $-.327^{\dagger}$ & 1 & & & & & & & & & \\
\hline CSA-R & -.125 & $.297^{*}$ & 1 & & & & & & & & \\
\hline MMDL & $-.428^{\dagger}$ & $.536^{\dagger}$ & .077 & 1 & & & & & & & \\
\hline MMDA & $.402^{\dagger}$ & -.185 & -.120 & $-.431^{\dagger}$ & 1 & & & & & & \\
\hline MSDL & -.205 & $.458^{\dagger}$ & .150 & $.681^{\dagger}$ & -.104 & 1 & & & & & \\
\hline MSDA & $.353^{\dagger}$ & $-.280^{*}$ & $-.319^{\dagger}$ & $-.604^{\dagger}$ & $.531^{\dagger}$ & $-.627^{\dagger}$ & 1 & & & & \\
\hline SCV & $.240^{*}$ & $-.391^{\dagger}$ & -.061 & $-.626^{\dagger}$ & .130 & $-.816^{\dagger}$ & $.578^{\dagger}$ & 1 & & & \\
\hline $\begin{array}{l}\text { MUAP } \\
\text { amplitude }\end{array}$ & $-.255^{*}$ & .032 & -.027 & .168 & -.097 & .061 & -.067 & -.089 & 1 & & \\
\hline $\begin{array}{l}\text { MUAP } \\
\text { duration }\end{array}$ & $-.273^{*}$ & .157 & $.318^{+}$ & $.282^{*}$ & -.203 & .166 & -.145 & -.130 & $.310^{\dagger}$ & 1 & \\
\hline BCTQ & $-.483^{\dagger}$ & .215 & $.296^{*}$ & $.263^{*}$ & -.159 & .203 & $-.308^{\dagger}$ & -.182 & $.351^{\dagger}$ & $.339^{\dagger}$ & 1 \\
\hline Severity & $-.364^{\dagger}$ & $.407^{\dagger}$ & .022 & $.801^{\dagger}$ & $-.508^{\dagger}$ & $.649^{\dagger}$ & $-.619^{\dagger}$ & $-.644^{\dagger}$ & $.244^{*}$ & .222 & $.225^{*}$ \\
\hline
\end{tabular}

CSA-P: Cross-sectional area of median nerve at pisiform level, CSA-R: Cross-sectional area of median nerve at distal radioulnar joint level, MMDL: Median motor distal latency, MMDA: Median motor distal amplitude, MDSL: Median sensory distal latency, MSDA: Median sensory distal amplitude, SCV: Sensory nerve conduction velocity at wrist segment of median nerve, BCTQ: The score of Boston carpal tunnel questionnaire, Severity: Electrophysiologic severity

${ }^{*} \mathrm{p}<0.05,{ }^{\dagger} \mathrm{p}<0.01$

Amplitude of MUAP showed statistically significant positive correlation with duration of MUAP $(r=0.310$, $\mathrm{p}=0.006)$, total score of BCTQ ( $\mathrm{r}=0.351, \mathrm{p}=0.002)$. Duration of MUAP showed statistically significant positive correlation with CSA of median nerve at distal radioulnar joint level $(\mathrm{r}=0.318, \mathrm{p}=0.008)$, distal latency of CMAP ( $\mathrm{r}=0.282, \mathrm{p}=0.012)$, and total score of BCTQ $(\mathrm{r}=0.339, \mathrm{p}=0.002)$, respectively (Table 2$)$.

\section{DISCUSSION}

The motor unit is the basic functional unit of the 
peripheral nervous system for movement and is a physiological unit that refers to anterior horn cells, axon and, myelin of the nerve, neuromuscular junction and all affiliated muscle fibers. In 1971, McComas et al. ${ }^{5}$ was the first to utilize the manual incremental method for MUNE and many investigators have suggested methods for MUNE measurement. ${ }^{16,17}$ The manual incremental method utilized in our investigation using nerve conduction study, which is easy as patient cooperation is not required unlike the spike-triggered averaging technique $^{16}$ that requires the subject to voluntarily contract muscles. Also, this method has the advantage of requiring a shorter time than multiple point stimulation ${ }^{17}$ and statistical method. ${ }^{3,18}$ In our clinic, 10-20 min were required for the MUNE examination and none of subjects complained discomfort or pain.

MUNE is used to assess motor unit functioning in a quantitative manner and is a sensitive method for the early diagnosis and evaluation of the severity and progression rates, and predicting survival rates of amyotrophic lateral sclerosis (ALS). MUNE is helpful in predicting the natural progression of ALS or therapeutic response or setting future therapeutic plans. ${ }^{19,20}$

In the early stages of motor unit loss, the amplitude of CMAP is decreased but with regeneration in chronic lesions by progressive compensatory collateral sprouting, the estimation of motor unit loss with CMAP amplitude alone is difficult. MUNE has the theoretic advantages of measuring the severity of nerve injury in neuropathy with retained CMAP amplitude. In our study, median motor distal amplitude and MUNE represent axonal loss that correlated significantly with each other. However, patients with low MUNE and normal range of median motor distal amplitude are observed commonly, while patients with a normal range MUNE and low median motor distal amplitude are not observed. Accordingly, continuous collateral sprouting in the remaining axons in CTS patients could be assumed. Bayrak et al. ${ }^{21}$ reported that all patients with low MUNE and maintain CMAP amplitude were severe CTS patient group in stage 4 of electrophysiologic severity, however our results showed these patients to be in all stages. In these cases, it was thought that careful caution were required to the diagnosis and treatment of CTS due to the few motor unit numbers leading to vulnerable to further progression to CTS. Studies on prognosis or therapeutic effect according to MUNE were not carried out and further research on MUNE as a useful diagnostic method to estimate prognosis and therapeutic response such as in ALS is needed.

In 1973, Brown ${ }^{4}$ was the first to utilize MUNE in reporting the decrease of motor units in 19 patients suspected of CTS and still decrease of MUNE in patients with recovered muscular strength by functional compensation through the reorganization of motor units from axonal degeneration. In 2000, Cuturic and Palliyath ${ }^{10}$ reported that MUNE measured by statistical methods in CTS patients diagnosed by electrodiagnostic studies had a decreased mean value in comparison to the control group, but this was not statistically significant. However, Bayrak et al. ${ }^{7,21}$ and KoÇ et al. ${ }^{6}$ later reported that MUNE decreased in CTS patients and our study also confirmed these findings.

A study on the measurement of differences in MUNE according to severity of CTS by Bayrak et al. ${ }^{7,21}$ revealed the statistically significant correlation between MUNE and electrophysiologic severity, latency and amplitude of CMAP, CSA of median nerve and our study showed same results. Also in this research, MUNE was significantly correlated with sensory nerve conduction velocity at carpal tunnel, amplitude of SNAP of median nerve, amplitude and duration of MUAP of APB and clinical scales of CTS. Thus, MUNE could be suggested as a useful examination test that reflects the change in sensory nerves, as well as in motor nerves of the CTS and reflect the severity of CTS.

Quantitative MUAP analysis or interference pattern analysis are useful examinations for diagnosing myopathy or neuropathy in neuromuscular disorders. As reinnervation proceeds following peripheral nerve injury, the amplitude and duration of MUAP increases and the interference pattern decreases. This was confirmed in our study as the amplitude and duration of MUAP of APB in the CTS group increased over the control group. And the area under the ROC curves of MUAP amplitude and duration are all more than 0.9 , suggesting that MUAP amplitude and duration measurement is a useful method in CTS diagnosis. But Cho et al. ${ }^{22}$ described that the mean amplitude and envelop amplitude, number of small segments in quantitative electromyographic study showed no statistical difference between CTS and control groups. Accordingly, further research is 
needed to evaluate the diagnostic utility of quantitative electromyographic study in CTS.

Werner and Albers ${ }^{11}$ reported delayed distal latency and decreased amplitude of median motor and sensory nerves as MUAP amplitude and duration increases and the degree of abnormal spontaneous activities increases. This research revealed the tendency for delayed distal latency and decreased amplitude of median motor and sensory nerves, as MUAP amplitude and duration increases, but only the MUAP duration and distal latency of CMAP were statistically significant. MUAP amplitude increased as electrophysiologic severity increased with maximal value at Padua stage 3 , while MUAP duration showed no statistically significant differences according to Padua stages. It has been reported that MUAP amplitude constantly increases with progression of reinnervation after peripheral nerve injury. ${ }^{23,24}$ However, in this research, MUAP amplitude increased with progression of CTS and decreased when reaching a stage of severe nerve damage. The cause was thought to be due to reduction in innervated muscle fibers, fibrosis or muscle atrophy from severe nerve degeneration.

The median nerve of CTS patients have increased CSA measured from ultrasonography at pisiform and distal radioulnar joint level due to edema of median nerve in the proximal carpal tunnel. ${ }^{8,25}$ In this research, CSA of median nerve was increased in both pisiform and distal radioulnar level with further aggravating electrophysiological severity. In particularly, the CSA at the pisiform level in the Padua stages 4 and 5 was significantly increased in comparison to stage 1 . Also, the median nerve CSA showed significant correlation with nerve conduction test results, such as motor nerve distal latency, sensory nerve distal latency and amplitude, and sensory nerve conduction velocity. Existing studies report controversial results of correlation between median nerve CSA, electrophysiological severity and symptom severity scales. ${ }^{8,26-28}$ Therefore, a more specialized and systematic research is deemed necessary.

This study utilized the latency difference in SNAP of the second finger between at wrist and palm stimulation for diagnosis of CTS. Park et al. ${ }^{29}$ reported a distoproximal ratio of median SNAP in the second and third digit as the most sensitive, but is a less specific parameter in the diagnosis of CTS; the combination of these parameters will be helpful in increasing the diagnostic sensitivity.
However, our investigation has the limitation of not analyzing the distoproximal ratio of median SNAP in the third finger.

\section{CONCLUSION}

CTS patients had a significant decrease in MUNE and an increase in amplitude and duration of MUAP of APB compared to the control group. MUNE showed a statistically significant correlation with NCS of median nerve, electrophysiologic severity, amplitude and duration of MUAP of APB, CSA of median nerve at pisiform level and clinical scales. MUAP amplitude of APB showed significant correlation with electrophysiologic severity and clinical scales, while MUAP duration of APB showed significant correlation with CSA of median nerve at distal radioulnar joint level and clinical scales, respectively.

Therefore, we suggested that the MUNE and quantitative MUAP analysis of APB are useful in diagnosis of CTS and, MUNE could be used as a sensitive test that well reflects the disease severity in CTS.

\section{REFERENCES}

1. Di Guglielmo G, Torrieri F, Repaci M, Uncini A. Conduction block and segmental velocities in carpal tunnel syndrome. Electroencephalogr Clin Neurophysiol 1997; 105: 321-327

2. Stevens JC. AAEM minimonograph \#26: the electrodiagnosis of carpal tunnel syndrome. American Association of Electrodiagnostic Medicine. Muscle Nerve 1997; 20: 1477-1486

3. Daube JR. Estimating the number of motor units in a muscle. J Clin Neurophysiol 1995; 12: 585-594

4. Brown WF. Thenar motor unit estimates in the carpal tunnel syndrome. J Neurol Neurosurg Psychiatry 1973; 36: 194-198

5. McComas AJ, Fawcett PR, Campbell MJ, Sica RE. Electrophysiological estimation of the number of motor units within a human muscle. J Neurol Neurosurg Psychiarty 1971; 34: 121-131

6. KoÇ F, Yerdelen D, Sarica Y, Sertdemir Y. Motor unit number estimation in cases with carpal tunnel syndrome. Int J Neurosci 2006; 116: 1263-1270

7. Bayrak IK, Bayrak AO, Tilki HE, Nural MS, Sunter T. Ultrasonography in carpal tunnel syndrome: com- 
parison with electrophysiological stage and motor unit number estimate. Muscle Nerve 2007; 35: 344-348

8. Pino LJ, Stashuk DW, Boe SG, Doherty TJ. Probabilistic muscle characterization using QEMG: application to neuropathic muscle. Muscle Nerve 2010; 41: 18-31

9. Stalberg E, Nandedkar SD, Sanders DB, Falck B. Quantitative motor unit potential analysis. J Clin Neurophysiol 1996; 13: 410-422

10. Cuturic M, Palliyath S. Motor unit number estimate (MUNE) testing in male patients with mild to moderate carpal tunnel syndrome. Electromyogr Clin Neurophysiol 2000; 40: 67-72

11. Werner RA, Albers JW. Relation between needle electromyography and nerve conduction studies in patients with carpal tunnel syndrome. Arch Phys Med Rehabil 1995; 76: 246-249

12. Dumitru D. Electrodiagnostic medicine, 2nd ed, Philadelphia: Hanley \& Belfus Inc, 2002, 1058-1070

13. Padua L, LoMonaco M, Gregori B, Valente EM, Padua R, Tonali P. Neurophysiological classification and sensitivity in 500 carpal tunnel syndrome hands. Acta Neurol Scand 1997; 96: 211-217

14. Stalberg E, Falck B, Sonoo M, Stalberg S, Astrom M. Multi-MUP EMG analysis - a two year experience in daily clinical work. Electroencephalogr Clin Neurophysiol 1995; 97: 145-154

15. Levine DW, Simmons BP, Koirs MJ, Daltroy LH, Hohl GG, Fossel AH, Katz JN. A self-administered questionnaire for the assessment of severity of symptoms and functional status in carpal tunnel syndrome. J Bone Joint Surg Am 1993; 75: 1585-1592

16. Bromberg MB. Motor unit estimation: reproducibility of the spike-triggered averaging technique in normal and ALS subjects. Muscle Nerve 1993; 16: 466-471

17. Doherty TJ, Brown WF. The estimated numbers and relative sizes of thenar motor units as selected by multiple point stimulation in young and older adults. Muscle Nerve 1993; 16: 355-366

18. Yune YS, Sohn MK, Kim BO. Motor unit number estimation of the thenar muscles. J Korean Acad Rehab Med 1997; 21: 1184-1193
19. Boe SG, Stashuk DW, Doherty TJ. Motor unit number estimates and quantitative motor unit analysis in healthy subjects and patients with amyotrophic lateral sclerosis. Muscle nerve 2007; 36: 62-70

20. Olney RK, Yuen EC, Engstrom JW. Statistical motor unit number estimation: reproducibility and sources of error in patients with amyotrophic lateral sclerosis. Muscle nerve 2000; 23: 193-197

21. Bayrak AO, Tilki HE, Coşkun M. Sympathetic skin response and axon count in carpal tunnel syndrome. J Clin Neurophysiol 2007; 24: 70-75

22. Cho SK, Park YK, Lee SC, Moon JH, Min KH, Park YB. Utility of quantitative electromyography in the evaluation of carpal tunnel syndrome. J Korean EMG Electrodiagn Med 2007; 9: 36-42

23. Miller RG. AAEM minimonograph \#28: injury to peripheral motor nerves. Muscle Nerve 1987; 10: 698710

24. Sohn MK, Yune YS. Changes of electromyographic signals following peripheral nerve injury. J Korean Acad Rehab Med 1997; 21: 547-552

25. Buchberger W. Radiologic imaging of the carpal tunnel. Eur J Radiol 1997; 25: 112-117

26. Visser LH, Smidt MH, Lee ML. High-resolution sonography versus EMG in the diagnosis of carpal tunnel syndrome. J Neurol Neurochir Psychiatry 2008; 79: $63-67$

27. Kaymak B, Ozçakar L, Cetin A, Candan Cetin M, Akinci A, Hasçelik Z. A comparison of the benefits of sonography and electrophysiologic measurements as predictors of symptom severity and functional status in patients with carpal tunnel syndrome. Arch Phys Med Rehabil 2008; 89: 743-748

28. Kele H, Verheggen R, Bittermann HJ, Reimers CD. The potential value of ultrasonography in the evaluation of carpal tunnel syndrome. Neurology 2003; 61: 389391

29. Park DS, Nam HS, Lee SE, Kim DH, Lee JG, Jeong HO. The usefulness of various electrodiagnostic parameters in mild carpal tunnel syndrome. J Korean EMG Electrodiagn Med 2008; 10: 135-143 
Appendix 1. Electrophysiologic Severity of Carpal Tunnel Syndrome (by Padua) ${ }^{13}$

Stage Criteria of findings

1 Abnormal segmental tests

2 Slowing of digit-wrist segment and normal distal motor latency (DML)

3 Slowing of median digit-wrist segment and abnormal DML

4 Absence of median SNAPs (digit-wrist segment) and abnormal DML

5 Absence of thenar motor (and sensory) response 